\title{
Adsorcion de xantato isopropilico de sodio (z-11) en carbon sub-bituminoso
}

\section{Adsorption of sodium isopropyl xanthate (z-11) on sub-bituminous carbono}

DOI: $10.46932 / \mathrm{sfjdv} 2 \mathrm{n} 5-140$

Received in: Oct 1st, 2021

Accepted in: Dec 30th, 2021

\section{Dra.Claudia Verónica Reyes Guzmán}

Facultad de Metalurgia U.A. de C

Apolonio m. avilés $411 \mathrm{col}$. Borja cp. $25680 \mathrm{~cd}$. frontera Coahuila México

E-mail: clavereyes@gmail.com / claudia_reyes_guzman@uadec.edu.mx

\section{Leonor Muñoz Ramírez}

M.C

Facultad de Metalurgia U.A. de C

Carretera 57 Km. 5 cp. 25710 Monclova Coahuila

E-mail: lemunozr@uadec.edu.mx

Dr. Sergio García Villarreal

Facultad de Metalurgia U.A. de C

Carretera 57 Km. 5 cp. 25710 Monclova Coahuila

E-mail: sergio_garcia@uadec.edu.mx

\section{Dr.Samuel Chacón de la Rosa}

C.I.G.A U.A. de C

5 de Febrero esq. con Blvd. Simón Bolívar \#303-A Col. Independencia C.P. 26830 Nueva Rosita, Coahuila, México.

E-mail: samuel_chacon@uadec.edu.mx

Gloria Guadalupe Treviño vera

M.C

Facultad de Metalurgia U.A. de C Carretera 57 Km. 5 cp. 25710 Monclova Coahuila

E-mail: gloriatrevinovera@uadec.edu.mx

\section{Dr.Diego Martínez Carrillo}

C.I.G.A U.A. de C

5 de Febrero esq. con Blvd. Simón Bolívar \#303-A Col. Independencia C.P. 26830 Nueva Rosita, Coahuila, México

E-mail: diegomartinez@uadec.edu.mx

\section{Dra. Isabel Araceli Facundo Arzola}

Facultad de Metalurgia U.A. de C

Carretera 57 Km. 5 cp. 25710 Monclova Coahuila

E-mail: isabelfacundo@uadec.edu.mx 


\title{
Ing. Darinka Daniela Rodriguez Sanchez
}

Exalumna Facultad de Metalurgia

U.A. de C

Carretera 57 Km. 5 cp. 25710 Monclova Coahuila

E-mail: darinkaDRodriguez@hotmail.com

\begin{abstract}
RESUMEN
La flotación es una técnica de limpieza de minerales sumamente importante, ya que se ha utilizado durante más de un siglo en la industria minera, para separar materiales valiosos de los no valiosos. Sin reactivos, no habría flotación, y sin el proceso de flotación, la minería tal como la conocemos hoy día, no existiría. Los reactivos de flotación promueven, intensifican y modifican las condiciones óptimas del mecanismo fisicoquímico de los minerales de flotación y pueden ser sustancias orgánicas e inorgánicas. La presente investigación surgió por la necesidad de reusar el xantato isopropílico de sodio que se desecha del proceso de flotación y adsorberlo en la superficie de un carbón de la región como es el carbón sub-bituminoso. Con el propósito de cuantificar la adsorción y desorción de xantato isopropílico de sodio (z-11) en la superficie del carbón, se analizaron las muestras por medio de diferentes técnicas de caracterización, XPS, Espectroscopia Raman y UV-Vis. En caracterizaciones por espectroscopia fotoelectronica de rayos x, se encontraron los principales grupos funcionales del carbón que interactúan con el xantato proveniente de las flotaciones. Estos fueron en su mayoría grupos carbonil y carboxil.
\end{abstract}

Palabras claves: z-11, carbón activado, espectroscopia fotoelectronica de rayos x.

\begin{abstract}
Flotation is an extremely important mineral cleaning technique that has been used for over a century in the mining industry to separate valuable from non-valuable materials. Without reagents, there would be no flotation, and without the flotation process, mining as we know it today would not exist. Flotation reagents promote, enhance and modify the optimum conditions of the physicochemical mechanism of flotation minerals and can be organic or inorganic substances. The present investigation arose from the need to reuse the sodium isopropyl xanthate that is discarded from the flotation process and adsorb it on the surface of a coal from the region such as sub-bituminous coal. In order to quantify the adsorption and desorption of sodium isopropyl xanthate (z-11) on the surface of the coal, the samples were analyzed by means of different characterization techniques, XPS, Raman Spectroscopy and UV-Vis. In characterizations by X-ray photoelectron spectroscopy, the main functional groups of the coal that interact with the xanthate coming from the flotation were found. These were mostly carbonyl and carboxyl groups.
\end{abstract}

Keywords: z-11, activated carbon, x-ray photoelectron spectroscopy.

\section{INTRODUCCION}

A Flotación con xantato isopropílico de sodio

La flotación es un proceso industrial de separación que utiliza las diferencias de propiedades superficiales entre el mineral valioso y el de poco interés económico, también conocido como ganga [1]. Específicamente, el proceso de separación selectiva toma ventaja de las diferencias de hidrofobicidad (o mojabilidad) existentes entre los diferentes minerales que componen el mineral original [2]. Desafortunadamente, pocas especies tales como talco, grafito, carbón, molibdenita, estibina y azufre 
poseen naturaleza hidrófoba significativa, en consecuencia, para lograr la separación selectiva de minerales que tienen un carácter más bien hidrófilo, es necesario proporcionar características hidrófobas a éstos, mediante la adición de un reactivo, conocido como colector [3].

Los colectores son compuestos de origen orgánico, que reducen la afinidad de las partículas por agua a través de la adsorción de moléculas o iones en la superficie del mineral, reduciendo la estabilidad de la capa hidratada, de forma de permitir la adhesión con las burbujas [4-5].Estos reactivos pueden ser iónicos y no iónicos. Los primeros son compuestos heteropolares que se disocian o ionizan en agua, y están formados por un grupo apolar (hidrocarburo) que se orienta hacia la fase líquida, sin interactuar con ésta, y un grupo polar que reacciona con la superficie del mineral (en los sitios activos) por medio de una fisisorción o quimisorción. Los colectores del tipo no iónicos no son solubles en agua, pero cumplen el mismo rol que los iónicos $[3,1]$.

Para lograr que la separación sea lo más eficiente posible, las burbujas de aire que ingresan al sistema deben ser estables y poseer un tamaño apropiado para realizar la colección del mineral. Para esto, se adicionan los reactivos conocidos como espumantes, que poseen estructura similar a los colectores iónicos (heteropolares), pero en este caso, el grupo polar presenta gran afinidad con el agua.

Otros reactivos que son útiles en el proceso de flotación son los modificadores o reguladores usados para variar la acción de un colector, intensificando o reduciendo su efecto en la hidrofobicidad de la superficie de los minerales, confiriendo mayor selectividad para las partículas de interés. Según la clasificación de Wills (2006) pueden ser clasificados en:

$\nabla$ Activadores: Estos reactivos alteran la naturaleza química de la superficie del mineral de forma de fortalecer el enlace entre la superficie y el colector, o aumentando la adsorción de estos últimos sobre la superficie del mineral. Los activadores son usualmente sales solubles que se ionizan en solución, luego los iones reaccionan con la superficie del mineral.

$\nabla$ Depresantes: Son usados para incrementar la selectividad de la flotación volviendo ciertos minerales hidrófilos previniendo por tanto su flotación.

$\boldsymbol{\nabla}$ Reguladores de $\mathrm{pH}$ : Reactivos que se utilizan para controlar las condiciones de $\mathrm{pH}$ que son determinantes en la adsorción de los colectores, varían en función del medio a utilizar (alcalino o ácido).

Los xantatos son ditiocarbonatos los cuales son utilizados como colectores en los procesos de flotación para la mayoría de los minerales sulfurados, así como elementos metálicos tales como el cobre, níquel, zinc, plomo, oro y plata.

El tipo de colector más utilizado en la actualidad es el xantato isopropílico de sodio. Sin embargo, después de éste cumplir su función y ser utilizados en el proceso de flotación, suelen ser descargados en las presas de jales donde además de permanecer como remanente tóxico, afectan el suelo, flora y fauna marina. [2] 
El impacto negativo del xantato al medio ambiente, en la vida acuática y de suelo se ha registrado en distintos aspectos. Se han estudiado los efectos tóxicos que los colectores de flotación tienen sobre la actividad microbial del suelo [3]. Asimismo, se han hecho estudios sobre peces y distintos tipos invertebrados [4] los cuales demuestran que después del primer día de remanencia del xantato como desecho, afecta de forma considerable la vida acuática. Así como los efectos de los productos de descomposición del xantato, tales como el dixantógeno [5-8]. Quienes entre otras cosas demostraron que para plantas acuáticas (lemna minor) la toxicidad de los xantatos causó una mortalidad del 100\% a una concentración menor a $5 \mathrm{mg} / \mathrm{L}$. [9]

Tomando en consideración la utilidad que ocupan los xantatos en la industria minera, y debido a la toxicidad que representan para el medio ambiente el uso de éstos reactivos, es necesario desarrollar una metodología de evaluación de xantatos en efluentes de procesos de flotación, así como la adsorción de los residuos de xantatos en los efluentes del proceso de flotación de minerales.

\section{B Carbón activado}

Tipos de carbón

El carbón en realidad es un sedimento mineral que contiene un alto porcentaje de carbono, un bajo porcentaje de humedad y un porcentaje muy pequeño de nitrógeno. El carbono cuando se quema en contacto con el aire o el oxígeno proporciona energía térmica, que es lo que después se transforma en otros tipos de energía. El carbón se categoriza según su dureza o su contenido de carbono:

$\nabla$ Grafito: carbono superior, pero difícil de quemar, por tanto, poco utilizado para combustión.

$\nabla$ Antracita: carbón de buena calidad, muy duro, a menudo utilizado para calentamiento.

$\nabla$ Carbón bituminoso: carbón denso que puede convertirse en coque, un combustible elaborado mediante la destilación de carbones más blandos; se usa para calentar los altos hornos de las centrales metalúrgicas.

$\boldsymbol{\nabla}$ Carbón sub-bituminoso: carbón con calidades del carbón bituminoso y el lignito.

$\boldsymbol{\nabla}$ Lignito: carbón de baja categoría con mucha humedad, pero que todavía ocupa un lugar en las centrales termoeléctricas.

El carbón bituminoso es el tipo más útil para el sector metalúrgico. Se combina con hierro y esta mezcla se calienta en hornos industriales a una temperatura de $1000{ }^{\circ} \mathrm{C}$. Este proceso reduce los óxidos del hierro y produce acero. El carbón también puede gasificarse en presencia de vapor de agua y oxígeno. El resultado es un gas sintético fabricado con monóxido de carbono e hidrógeno. Según la forma de convertirlo, puede convertirse en metanol, hidrógeno puro, combustible sintético, etc. 
Para muchas de estas aplicaciones se recurre al uso de carbón activado como material adsorbente, debido a sus reconocidas propiedades: área superficial muy grande, microporosidad elevada y económicamente de bajo costo, entre otras [10-11]

Después que se realiza la flotación los efluentes son mandados a la presa de jales y tirados a la intemperie, por ello surgió esta investigación con ayuda del poder adsorbente de los carbones activados, ponerlos en contacto con la solución de flotación y adsorber el xantato isopropílico de sodio para poderlo atrapar en la superficie del carbón y liberarlo para recircularlo al proceso.

\section{DESARROLLO EXPERIMENTAL}

\subsection{DETERMINACIÓN DE PUNTOS DE CARGA CERO P.C.C.}

Este método se basa en la estimación del punto de carga cero (PCC) de la superficie del carbón a partir de la medición del pH de una suspensión en la que progresivamente se incrementa la cantidad de sólido en suspensión. Los fundamentos y detalles de la técnica fueron reportados en nuestro grupo de trabajo por Hernández-Orta (2007). El procedimiento para medir el PCC del Carbón es el siguiente:

1. Se colocó $25 \mathrm{~mL}$ de agua desionizada en un tubo de plástico de $50 \mathrm{~mL}$.

2. Se midió el $\mathrm{pH}$ inicial del agua desionizada

3. Se agregó $0.2 \mathrm{~g}$ del carbón de un tamaño de partícula de malla $-40+60$ en el tubo que contiene los $25 \mathrm{~mL}$ de agua desionizada como se muestra en la Figura 1.

4. Sumergir el recipiente parcialmente en un baño a temperatura controlada para mantener el sistema a $25^{\circ} \mathrm{C}$.

5. Agitar el recipiente de forma manual periódicamente (3 - 4 veces al día).

6. Medir el $\mathrm{pH}$ después de $12 \mathrm{~h}$.

7. Inmediatamente después de medir el pH, hacer una nueva adición de muestra (cantidad indiada en paso 3).

8. Repetir cíclicamente los pasos del 3 al 7; hasta que se obtenga un comportamiento asintótico del $\mathrm{pH}$ o hasta que no se observen cambios significativos en el $\mathrm{pH}$.

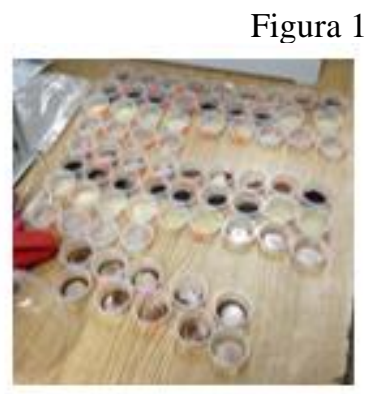

Muestras.

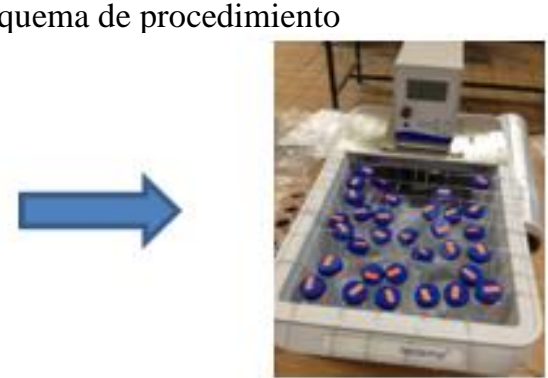

Baño de recirculación 


\subsection{MOLIENDA DE CARBÓN}

Primeramente, se tomaron muestras de carbón, con un peso total de $10 \mathrm{~g}$ c/u fue molida durante 30 min, 1 hora, 1.5 horas y 2 horas a una velocidad de $20 \mathrm{rpm}$, para observar cual era el mejor tiempo de molienda, con 0.5 horas de reposo por cada hora de trabajo. Esta experimentación fue realizada con la intención de encontrar el mejor tiempo de molienda.

Los componentes y las bolas de acero se cargaron a un vial de acero inoxidable, la molienda se realizó en un molino Restch para producir polvo de las diferentes muestras a tratar, el peso total de la muestra fue $10 \mathrm{~g}$.

En el proceso de molienda mecánica fue necesario, considerar 0.5 horas de reposo por cada hora de trabajo, lo cual fue debido al calentamiento del motor del molino. Una vez obtenida una gran cantidad de polvo del producto de las moliendas mecánicas, este se guardó en viales de vidrio con tapa para evitar cualquier contaminación.

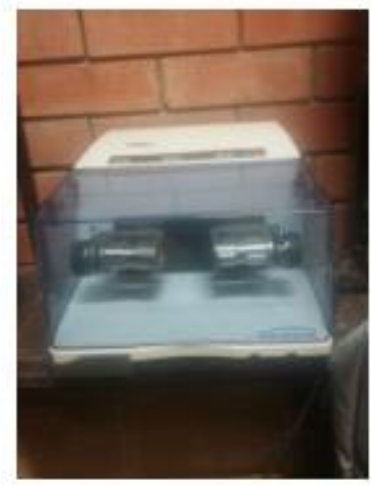

Molino.
Figura 2 Molino Restch.
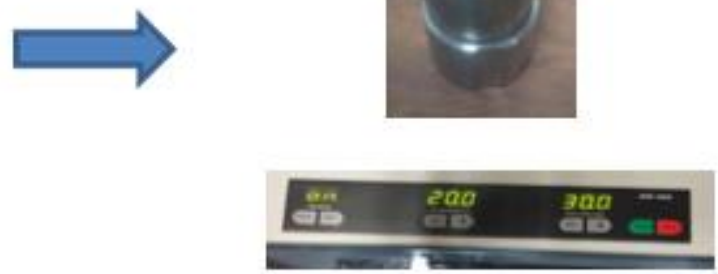

Vialy parámetros

\section{RESULTADOS Y DISCUSION}

La caracterización de espectroscopia uv-visible se le practicó un barrido a una longitud de onda de 200 a $400 \mathrm{~nm}$, dando como resultado, fue una recristalización del xantato como se muestra en las Figuras 3 mostrándose dos bandas máximas a 224 y 301, en 224 se cuantifica una especie llamada dixantogeno, a 301 se cuantifica el xantato, en nuestra investigación se cree que se formó la especie dixantogeno por la ayuda del carbón ya que una cantidad de xantato fue adsorbida por este y la siguiente banda fue producto de la descomposición u oxidación del xantato en la superficie. 
Figura 3.- Xantato isopropilico de sodio (z-11) a $301 \mathrm{~nm}$ y dixantogeno a $224 \mathrm{~nm}$

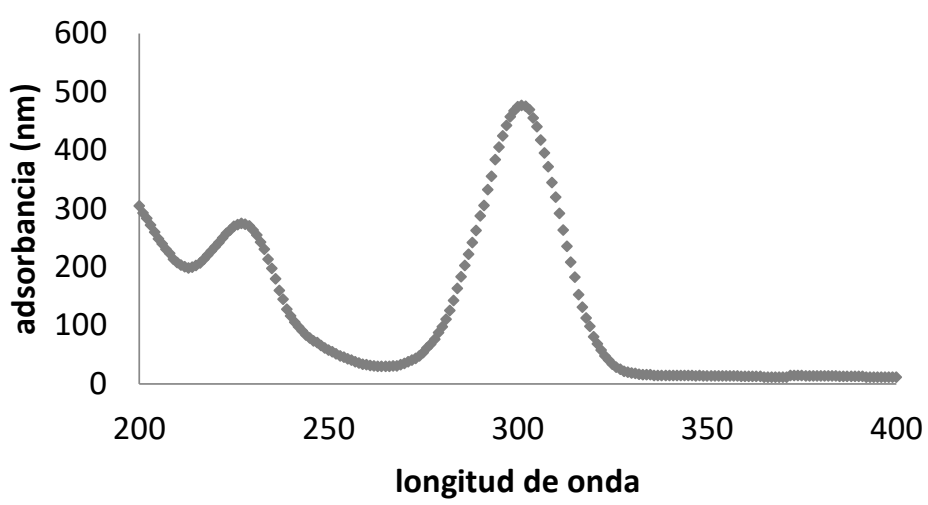

El espectro Raman de XIPNa adsorbido en la superficie del carbón se muestra en la figura 4. Se puede observar los distintos picos en los que se presenta el xantato isopropílico de sodio, el cual fue adsorbido en la superficie. El rango en el cual fueron analizadas las muestras fue desde 100 hasta 3500 $\mathrm{cm}^{-1}$ Los picos característicos del xantato aparecen entre las siguientes frecuencias 650, 1020, 1450-1500, y entre $2800-3000 \mathrm{~cm}^{-1}$

Figura 4. Espectro Raman de XIPNa

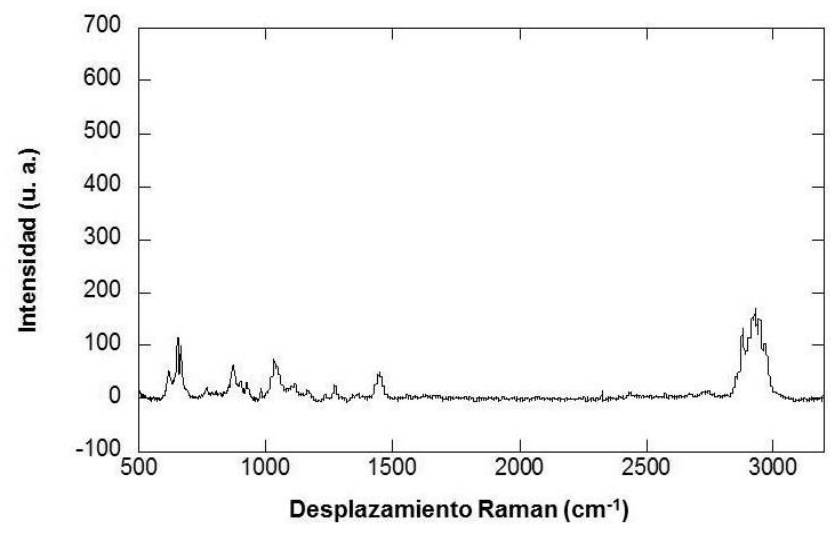

Otra técnica de caracterización que también se llevó a cabo fue la Espectroscopia de Fotoelectrones emitidos por Rayos X (XPS), con la cual se analizó la adsorción de xantato. Los espectros con mayor cantidad de xantato adsorbido, fueron analizados y comparados con la literatura correspondiente.

En base a estudios de xantato isopropilico de sodio que se han publicado anteriormente, según (K. C. Pillai, 1983), podemos comparar que, los resultados obtenidos en los espectros , mismos a los que no se realizó deconvoluciónes ya que se comparó con la literatura, muestran los picos característicos de la presencia de xantato en su superficie, los cuales producen corrimientos de los picos .

En la figura 5 se muestra el espectro del carbono (C) del nivel 1s en el cual se pueden observar 3 diferentes picos correspondientes a distintos compuestos enlazados con carbono. Para este espectro se 
define que existen 3 átomos de carbono químicamente no equivalentes localizados a 284.9, 286.3 y 288.1 $\mathrm{eV}$. Por este motivo podemos asumir que estos enlaces son correspondientes a enlaces xantato

Figura 5. Espectro muestra $1:$ nivel 1s del C.

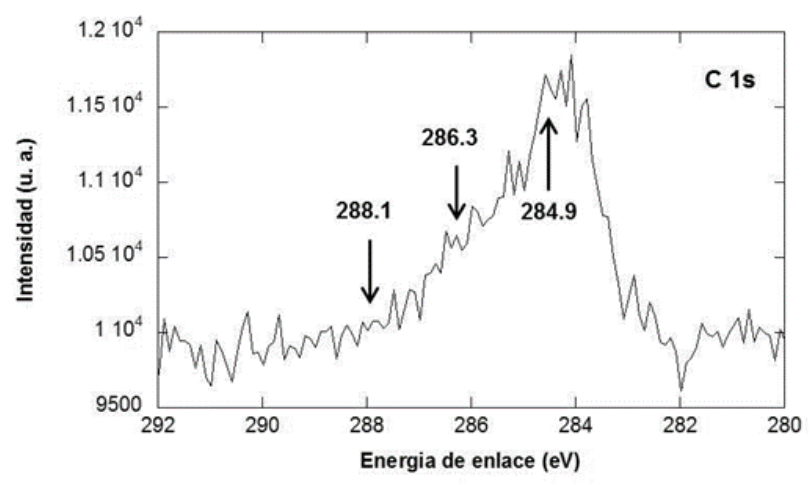

Las propiedades de textura del carbón (área específica, volumen de los poros y diámetro promedio de los poros), se determinaron por medio de un equipo de fisisorción de $\mathrm{N}_{2}$ y dio como resultado la Tabla IV.

Tabla I Área superficial especifica del carbón vegetal

\begin{tabular}{|l|l|}
\hline Carbón & S BET $\left(\mathbf{m}^{2} / \mathbf{g}\right)$ \\
\hline $\begin{array}{l}\text { Carbón mineral sub- } \\
\text { bituminoso }\end{array}$ & 900.87 \\
\hline Carbón vegetal & 500.87 \\
\hline
\end{tabular}

Este método se basa en la estimación del punto de carga cero (PCC) de la superficie del carbón a partir de la medición del pH de una suspensión en la que progresivamente se incrementa la cantidad de sólido en suspensión.

En la tabla V se obtuvieron los P.C.C de los carbones utilizados en la experimentación.

Tabla V.- P.C.C de los carbones

\begin{tabular}{|c|c|}
\hline CARBON & P.C.C \\
\hline 1 & 2.298 \\
\hline 2 & 13.96 \\
\hline
\end{tabular}

En numero 1 es el carbón sub-bituminoso con un punto de carga menor que la solución de xantato (pH 8), y el fenómeno que predomina es atracción electrostáticamente, el xantato con el carbón

\section{CONCLUSIONES}

De los espectros obtenidos por XPS y Raman determinamos que el xantato se adsorbio y desorbio de la superficie del carbon 
El xantato residual presente en los procesos de flotacion, puede ser removido utilizando en el carbon como adsorbente del mismo, esta tecnica desplaza otro tipo de tecnicas las cuales descomponen el xantato, ya que, para aplicar tecnologias encargadas de la descomposicion quimica, se requiere gran consumo de energia reflejado en elevados costos de operación.

El empleo de carbon como adsorbente beneficia sustancialmente, debido a que el xantato puede ser desorbido y retornado nuevamente al proceso aplicando una ligera purificacion del mismo. 


\section{BIBLIOGRAFIA}

1. Wills, B.A. Mineral Processing Technology. Edtion ed.: Elsevier Science \& Technology, 2006

2. Gaudin, A.M. Flotation: A.M. Gaudin memorial volume. edited by M. AMERICAN INSTITUTE OF MINING, AND PETROLEUM ENGINEERS. Edtion ed., 1976.

3. Leja, J. Surface Chemistry of froth flotation. Edtion ed., 2004.

4. Fuerstenau, M.C. Froth Flotation a century of innovation. edited by C.S.F.M. LITTLETON, METALLURGY, AND EXPLORATION. Edtion ed., 2007.

5. Nguyen, A.V. Colloidal Science of Flotation. edited by M. DEKKER. Edtion ed., 2004.

6. Cheng H., L. H., Huo H., Dong, Y. Xue, Q. Cao L. Continuous removal of ore flotation reagents by an anaerobic-aerobicbiological filter. Bioresource Technology, 255-261. 2012.

7. Chockalingam E., S. S., Natarajan K.A. Studies on biodegradation of organic flotation collectors using Bacillus polymyxa. Hydrometallurgy. 2003.

8. Gerardo Cifuentes Molina, C. H. C., Marco Antônio Siqueira Rodrigues, Andréa Moura Bernardes. Sodium isopropyl xanthate degradation by advanced oxidation processes. Minerals Engineering. 2013.

9. Howe, T. M., Pope, M.I. The quantitative determination of flotation agents adsorbed on mineral powders, using differential thermal analysis. Powder Technology. 1970/1971.

10. Podkoscielny, P., Nieszporek, K., Heterogeneity of activated carbons in adsorption of phenols from aqueous solutions - Comparison of experimental isotherm data and simulation predictions, Applied Surface Science, 253, 3563-3570, 2007.

11. Figueiredo, J. L., Pereira, M. F. R., Freitas, M. M. A., Órfão, J. J. M., Modification of the surface chemistry of activated carbons, Carbon, 37, 1379-1389, 1999

12. K. C. Pillai, V. Y. Y., J.OM. Bockris. XPS studies of Xanthate adsorption on galena surfaces. Applications of surface Science. 1983. 